\title{
CRT-dependent artifacts in contrast-sensitivity measurements
}

\author{
JOHN TRIMBLE and CHARLES KAMPSCHOER \\ Rehabilitation Research and Development Center, Hines, Illinois
}

\begin{abstract}
Previous studies have suggested that the physical dimensions of cathode-ray-tube display beams can have potentially adverse effects on the contrast of sinusoidal gratings, especially at high spatial frequencies. These effects are obviously important, because they could cause errors in contrastsensitivity measurements. Measures of these effects indicate that they can be predicted from the intensity profile of the display beam. Also, the half amplitude width of the beam's intensity profile limits the contrast of sinusoidal gratings at high spatial frequencies. This limitation could seriously affect contrast-sensitivity measurements at spatial frequencies as low as three cycles per degree, depending on viewing conditions.
\end{abstract}

Contrast sensitivity is usually measured with stimuli that are vertical, sinusoidal gratings displayed on a cathode ray tube (CRT). Knowing the contrast of these gratings is imperative for obtaining accurate results. However, the display's contrast can be affected by physical limitations of the CRT. Although theoretical calculations of these effects have been previously reported (Hess \& Baker, 1984; Morgan \& Watt, 1982), they have not been empirically measured. We measured how the contrast of sinusoidal gratings changes with spatial frequency and related these changes to the physical dimensions of the display beam.

The relationship between the contrast of a sinusoidal grating and the dimensions of the display beam is best understood by considering the way in which sinusoidal gratings are produced. Most investigators produce sinusoidal gratings by combining three waveforms, as shown in Figure 1: (A) a triangular waveform, which modulates the vertical position of the CRT beam; (B) a sawtooth waveform, which modulates the beam's horizontal position; and (C) a sinusoidal waveform, which modulates the beam's intensity (Schade, 1956). The ratio of the vertical and horizontal modulation frequencies establishes the minimum distance in which the intensity of the beam can change. For example, if the vertical modulation frequency $\left(f_{v}\right)$ is $1.0 \mathrm{MHz}$, and the horizontal modulation frequency $\left(f_{h}\right)$ is $100 \mathrm{~Hz}$, then the number of times the beam crosses an arbitrary point on the CRT is

$$
N=\frac{2 f_{v}}{f_{h}}=\frac{2 \times 10^{6}}{10^{2}}=2 \times 10^{4}
$$

If the width of the face of the CRT is $100 \mathrm{~mm}$, then the

This research was supported in part by funds from the United States Veterans Administration, Rehabilitation Research and Development Service. We thank Christina Enroth, Sidney Lehky, Gregory Phillips, and John Troy for their helpful comments. We also thank Cynthia Neubecker for her assistance in developing computer software. Our mailing address is: Rehabilitation Research and Development Center, VA Hines Hospital, Hines, IL 60141.
(A)

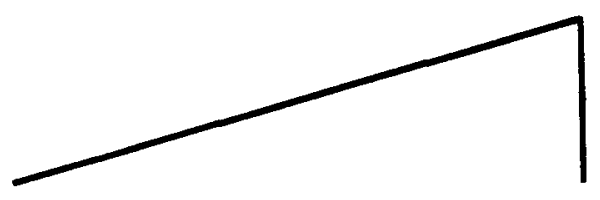

(B)

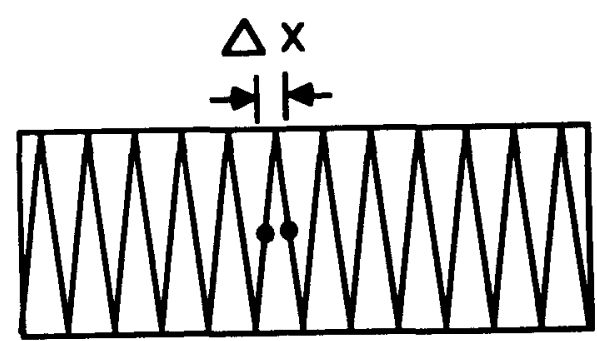

(C)

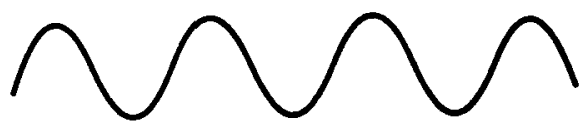

Figure 1. Waveforms used to produce vertical, one-dimensional sinusoidal gratings: (A) sawtooth waveform used to control the horizontal position of the beam; (B) triangular waveform used to control the vertical position of the beam; and $(C)$ sinusoidal waveform used to modulate the beam's intensity. $\Delta x$ is the horizontal distance traveled by the beam (closed circles) in one-half period of the vertical modulating waveform.

distance traveled by the beam in each half cycle of the vertical modulating waveform is

$$
\Delta x=\frac{100}{2 \times 10^{4}}=0.005 \mathrm{~mm},
$$

as shown in Figure 1. If $\Delta x$ is the period of a spatial waveform sampling the sinusoidal grating, then the contrast of the grating should not be adversely affected until its 
spatial frequency exceeds the Nyquist sampling rate (e.g., $1 / 2 \Delta x$ ), which is 400 cycles $/ \mathrm{mm}$ in this example.

However, in most cases, the CRT beam diameter is much greater than the figure given in Equation 2. Accordingly, the beam diameter can have a significant effect on the contrast of the grating. Our results show this effect, and provide a way to predict it from the half amplitude width of the beam-intensity profile.

\section{METHOD}

Vertical sinusoidal gratings were produced on a Tektronix 606A high-resolution display monitor using the programmable pattern generator shown in Figure 2. The pattern generator has a read-only memory (ROM) that contains 256 samples of one cycle of a sinusoidal function. These samples are transferred from the ROM to a multiplying digital-to-analog converter, whose output modulates the intensity of the CRT. A digitally programmable, variable-frequency clock oscillator controls the rate at which the samples are transferred. This rate controls the spatial frequency of the sinusoidal grating, since it determines the number of cycles that are contained in one period of the horizontal sweep of the display monitor. The contrast of the grating is controlled by the voltage applied to the multiplying input of the digital-to-analog converter.

The CRT's horizontal axis was controlled by its internal time base, which was set for a sweep period of $12.8 \mathrm{msec}$. The internal time base was triggered with a pulse produced by the programmable pattern generator to ensure synchrony between the horizontal sweep and the intensity-modulating waveform. The CRT's vertical axis was driven by a triangular waveform at frequency of $750 \mathrm{kHz}$. The vertical axis waveform was not synchronized with either the horizontal sweep or the intensitymodulating waveform.

The intensity profile of the CRT was measured using the video analyzer shown schematically in Figure 3 . The CRT screen was converted to a video image using a highresolution video camera (Cohu 5222) fitted with a high-

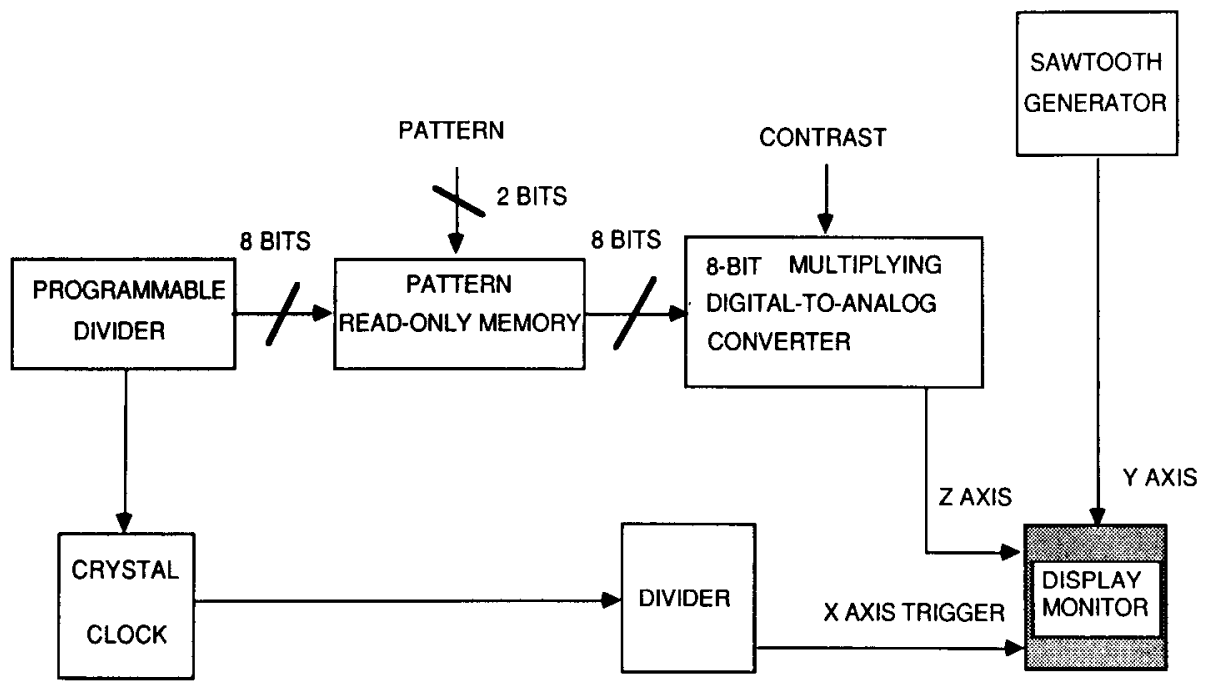

Figure 2. Block diagram for the programmable pattern generator.

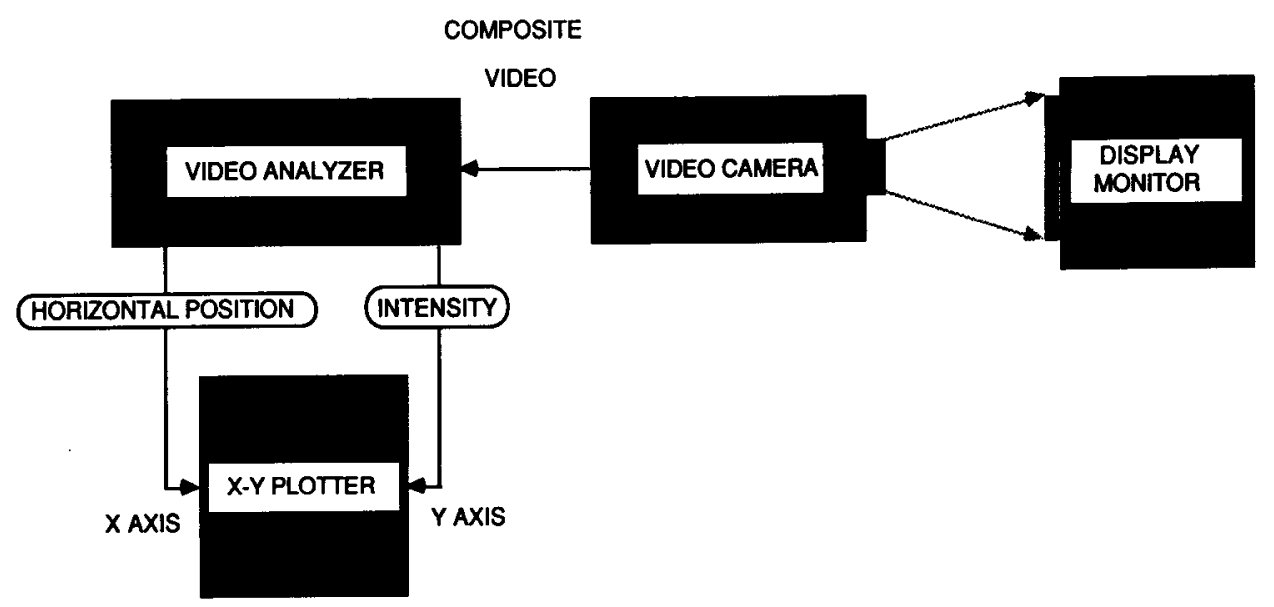

Figure 3. Block diagram for the video analysis system. 
quality lens (Nikon 55-mm MicroNikkor). The video signal from the camera was transferred to a video analyzer (Colorado Video Model 321), which provided an intensity profile of a selected portion of the video image. This profile was plotted on an X-Y recorder (Hewlett Packard 7015B) for subsequent analysis.

Measurements of the intensity profile were made at three camera-to-monitor distances to ensure that there were no errors caused by the resolution limits of the video camera or the optics. Representative samples of intensity profiles at three different spatial frequencies, and two different camera-to-monitor distances are shown in Figure 4 . These results clearly show that the measuring system did not affect our results.

The CRT beam's intensity profile was also measured with the video analyzer by disconnecting the horizontal modulating waveform to produce a single, vertical line in the center of the display.

The CRT's attenuation characteristics were determined by measuring the peak-to-peak amplitude of the intensity profile at each spatial frequency. The average of 20 measures was used to compute the grating's contrast.

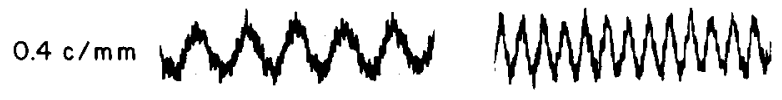

$0.8 \mathrm{c} / \mathrm{mm}$ WhWMWh

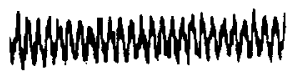

$1.0 \mathrm{c} / \mathrm{mm}$ WhWWHWWM

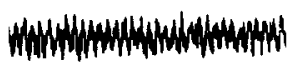

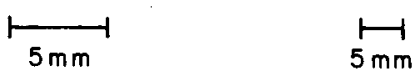

Figure 4. Sample intensity profiles at two measurement distances of sinusoidal gratings with three different spatial frequencies. The measurement distance was $57 \mathrm{~cm}$ for the profiles on the right and $28 \mathrm{~cm}$ for the profiles on the left.

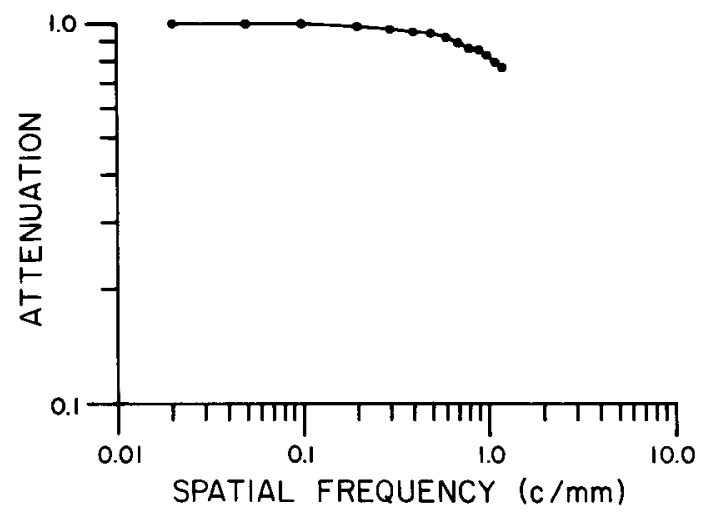

Figure 5. Attenuation characteristics of the CRT's z-axis electronics. Relative attenuation is the ratio between the z-axis modulation voltage and the CRT grid modulation voltage expressed as a fraction of the maximum voltage over the measurement range.

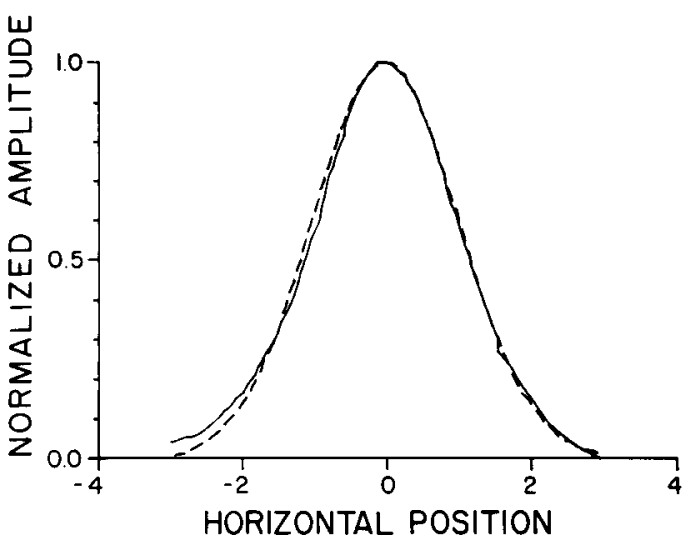

Figure 6. A comparison of the intensity profile of the CRT beam to the Gaussian function given by Equation 3.

The relative attenuation was computed from the ratio of the peak-to-peak amplitude at a given spatial frequency to the maximum peak-to-peak amplitude over the entire range of spatial frequencies.

We also measured the attenuation characteristics of the CRT's z-axis electronics to ensure that our results were not caused by frequency-dependent limitations in the intensity-modulating amplifiers. As shown in Figure 5, there is a slight reduction in the $\mathrm{z}$-axis grid modulation voltage when high spatial frequency gratings are displayed. We scaled our measures of the CRT's contrast to compensate for these characteristics.

\section{RESULTS}

The empirical intensity profile of the CRT beam is shown in Figure 6. It can be described by a Gaussian function of the form:

$$
h(x)=\frac{1}{\sigma \sqrt{2 \pi}} e^{-x^{2} / 2 \sigma^{2}},
$$

where $h(x)$ has a mean of zero and a variance of $\sigma^{2}$. Equation 3 provides an excellent fit to the empirical data if $\sigma$ is set to the half amplitude width of the intensity profile $(1.0 \mathrm{~mm})$, as shown in Figure 6.

If we assume that $h(x)$ is the impulse response of a linear spatial filter acting on the sinusoidal grating, then we can predict its effect on the grating by Fourier techniques (Papoulis, 1962). The Fourier transform of $h(x)$ is

$$
\begin{aligned}
H(\omega) & =\int_{0}^{\infty} h(x) e^{-j \omega x} d x \\
& =\sqrt{\pi / \sigma} e^{-\omega^{2} / 2 \sigma^{2}},
\end{aligned}
$$

where $\omega$ is spatial frequency in cycles/radian, and $\sigma$ is the standard deviation of $h(x)$ (Papoulis, 1962). According to Fourier theory, Equation 4 should accurately predict the empirical attenuation curve. The function provides an excellent fit to our empirical data, as shown in Figure 7. 


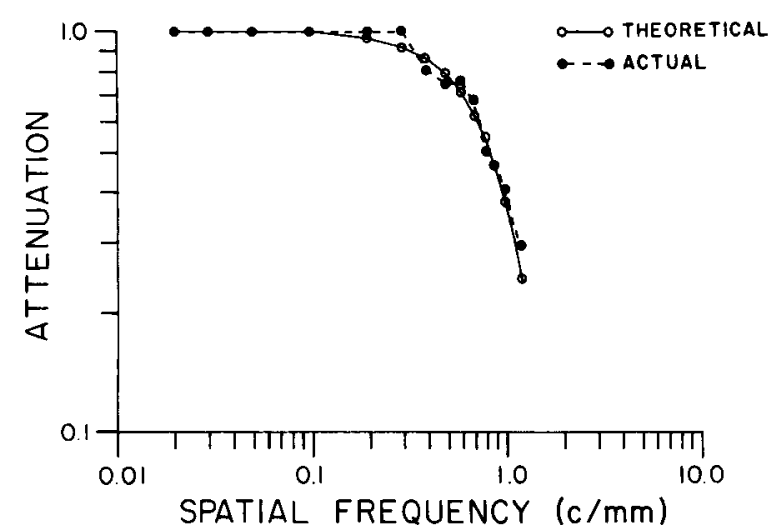

Figure 7. A comparison between the empirical and theoretical spatial attenuation characteristics of the CRT.

\section{DISCUSSION}

Our results not only confirm the theoretical predictions made by Morgan and Watt (1982), but they also offer an explanation of the primary factor limiting the contrast of sinusoidal gratings. Referring back to Figure 1, the diameter of the CRT beam is the factor limiting the resolution of the sinusoidal grating if the diameter of the CRT beam is much greater than the sampling period implied by the horizontal and vertical modulation frequencies. It is important to note that the beam diameter listed in the technical specifications for the CRT may be misleading, since other factors such as beam intensity and focus can affect it. Accordingly, complete confidence in the contrast of the sinusoidal grating can be obtained only if the beam's intensity profile has been determined empirically.

Our results suggest that considerable caution should be exercised in interpreting measures of contrast sensitivity made at high spatial frequencies. The extent to which the spatial attenuation characteristics of the display monitor will affect these measures depends entirely on the intensity profile of the CRT beam and the distance at which the CRT is viewed. For example, at a viewing distance of $57 \mathrm{~cm}$, the resolution characteristics of our display monitor will affect measures of contrast sensitivity above 3 cycles per degree (cpd). Referring to Figure 7, the actual contrast of the grating at $10 \mathrm{cpd}(1.0 \mathrm{cycle} / \mathrm{mm}$ with a viewing distance of $57 \mathrm{~cm}$ ) is $75 \%$ less than expected because of the physical limitations of the display. Accordingly, the threshold contrast at this spatial frequency will be $75 \%$ greater than actually measured. Clearly, there will be a significant error in the measurement of threshold contrast at this spatial frequency, unless these effects are considered. Thus, regardless of the type of CRT used for measuring contrast sensitivity, or the viewing distance used for measurements, it is important to know how the CRT's beam dimensions affect its resolution to obtain accurate contrast-sensitivity measurements.

\section{REFERENCES}

Hess, R. F., \& BAKER, C. L. (1984). Human pattern evoked electroretinogram. Journal of Neurophysiology, 51, 939-951.

MorGaN, M. J., \& WATT, R. J. (1982). The modulation transfer function of a display oscilloscope: Measurements and comments. Vision Research, 22, 1083-1085.

Papoulss, A. (1962). The Fourier integral and its applications. New York: McGraw-Hill.

SCHADE, O. H. (1956). Optical and photoelectric analog of the eye. Journal of the Optical Society of America, 46, 721-729.

(Manuscript received October 17, 1985; revision accepted for publication February 4, 1986.) 